J. Lake Sci. (湖泊科学), 2013, 25(1): 147-155

http: //www. jlakes. org. E-mail : jlakes@niglas.ac.cn

(c) 2013 by Journal of Lake Sciences

\title{
长江流域清江胡家溪四种蜉蝣优势种的周年生产量及其动态
}

\author{
江晶,温芳妮,王利肖,邱爽, 李晓宇,间云君** \\ (华中科技大学生命科学与技术学院,武汉 430074)
}

\begin{abstract}
摘 要: 2006 年 4 月至 2007 年 3 月对长江支流清江二级支流——胡家溪的大型底栖动物群落结构和生产量进行为期一 周年的调查和研究. 结果表明, 主要蜉蝣优势种小裳蜉 (Leptophlebia sp.) 、扁蜉 (Electrogena sp.) 、四节蜉 (Indobaetis sp.) 的生活史为一年三代, 细蜉 (Caenis sp.) 为一年两代; 小裳蜉的年均密度和年均生物量分别为 $407 \mathrm{ind} . / \mathrm{m}^{2} 、 1.00 \mathrm{~g} / \mathrm{m}^{2}$; 扁 蜉为 150 ind. $/ \mathrm{m}^{2} 、 0.37 \mathrm{~g} / \mathrm{m}^{2}$, 四节蜉为 232 ind. $/ \mathrm{m}^{2} 、 0.30 \mathrm{~g} / \mathrm{m}^{2}$, 细蜉为 91 ind. $/ \mathrm{m}^{2} 、 0.17 \mathrm{~g} / \mathrm{m}^{2}$. 采用龄期频率法测算的 周年生产量和 $P / B$ 分别为: 小裳蜉为 $441.42 \mathrm{~g} / \mathrm{m}^{2}$ (WW) , 14.3; 扁蜉为 $434.88 \mathrm{~g} / \mathrm{m}^{2}$ (WW) , 7.6 ; 四节蜉为 $747.21 \mathrm{~g} / \mathrm{m}^{2}$ (WW) 15.0 ; 细蜉为 $40.52 \mathrm{~g} / \mathrm{m}^{2}$ (WW) , 7.2. 四种蜉蝣生产量的时间重叠比例相似系数较高, 均大于 0.55 , 这可能与四种 蜉蝣的生境及食物资源较为相似有关.
\end{abstract}

关键词: 大型底栖动物; 群落; 生产量;动态;清江;蜉蝣; 胡家溪

\section{Annual production and its dynamics of four dominant mayflies in Hujiaxi Stream of Qingjiang River catchment, Yangtze River Basin}

\author{
JIANG Jing, WEN Fangni, WANG Lixiao, QIU Shuang, LI Xiaoyu \& YAN Yunjun \\ (College of Life and Science Technology, Huazhong University of Science and Technology, Wuhan 430074, P. R. China)
}

\begin{abstract}
During the period of April 2006 to March 2007, secondary production and its dynamics of the dominant species of mayfly community in a second order river ( Hujiaxi Stream) of Qingjiang River, Yangtze River Basin were investigated. From the upper to the lower reach of the stream, we sampled at six sites of different habitats quantitatively. The life cycles of the four dominant mayflies, namely Leptophlebia sp. , Electrogena sp., Indobaetis sp., and Caenis sp., were analyzed by the monthly size-class frequency distribution, the cohort and annual production were estimated by size frequency method, and the production dynamics were examined by the method sensu Benke and Wallace (1997). The results show that Leptophlebia sp., Electrogena sp. , and Indobaetis sp. appear to develop three generations per year, while Caenis sp. completed two generation a year. Their average annual standing stocks were: for Leptophlebia sp. , 407 ind. $/ \mathrm{m}^{2}, 1.00 \mathrm{~g} / \mathrm{m}^{2}$; for Electrogena sp. , 150 ind. $/ \mathrm{m}^{2}, 0.37 \mathrm{~g} / \mathrm{m}^{2}$; for Indobaetis sp. , 232 ind. $/ \mathrm{m}^{2}, 0.30 \mathrm{~g} / \mathrm{m}^{2}$; and for Caenis sp. , 91 ind. $/ \mathrm{m}^{2}, 0.17 \mathrm{~g} / \mathrm{m}^{2}$, respectively. The annual production $\left(\mathrm{g} / \mathrm{m}^{2}(\mathrm{WW})\right)$ and the annual $P / B$ ratio of four mayflies were: for Leptophlebia sp. , $441.42 \mathrm{~g} / \mathrm{m}^{2}$ (WW ), 14.3; for Electrogena sp. , $434.88 \mathrm{~g} / \mathrm{m}^{2}$ (WW) , 7.6; for Indobaetis sp. , 747.21 g/ $\mathrm{m}^{2}$ (WW), 15.0; and for Caenis sp. , $40.52 \mathrm{~g} / \mathrm{m}^{2}(\mathrm{WW}), 7.2$. Temporal overlap of secondary production dynamics of the four species, which measured with the proportional similarity index as 0.55 , indicates that they utilize similar or same food resources along time dimension.
\end{abstract}

Keywords: Macrozoobenthos; community; production; dynamic; Qingjiang River; mayfly; Hujiaxi Stream

大型底栖动物是河流生态系统中重要的生物类群之一, 能加速水底碎屑分解, 促进泥水界面物质交换 和水体自净, 是河流生态系统物质循环和能量代谢的重要参与者, 也是人们了解河流生态系统结构和功能 的关键类群之一. 此外, 由于底栖动物的生命周期相对较长、行动缓慢、物种丰富, 且不同种类底栖动物对环 境条件的适应性和对污染等不利因素的耐受力及敏感程度不同,因此作为重要的指示生物已广泛应用于水

* 国家自然科学基金项目 (30870427) 资助. 2011-04-13 收稿;2012-10-08 收修改稿. 江晶,女, 1984 年生,硕士研 究生;E-mail:jingjingiaiv@163.com.

** 通信作者;E-mail:yanyunjun@ tom. com. 
质及环境监测中, 起着水下哨兵的作用 ${ }^{[1]}$. 因此, 河流大型底栖动物群落结构与功能研究已成为生态学的热 点领域之一 ${ }^{[2]}$. 然而, 在我国, 河流生态学研究严重滞后, 与国际先进水平存在较大差距, 目前仅有少许对河 流功能研究的报道 ${ }^{[3-5]}$.

蜉蝣目昆虫广泛存在于各级河流中, 在河流生态系统中起着重要作用 ${ }^{[1-2]}$. 目前有关该类群的研究工 作多见于国外报道, 国内有关工作主要集中在分类学和水质监测方面 ${ }^{[6]}$, 而有关蜉蝣在河流生态系统中 的生态功能及营养动态则鲜见报道. 由于蜉蝣是河流生态系统食物链中极为重要的环节, 对维系群落 食物网的复杂性具有十分重要作用. 因此, 研究蜉蝣类群的生态学功能对了解和掌握我国河流生态系 统的结构与功能特点具有重要意义. 本文于 2006-2007 年对清江上游流域一二级河流 (胡家溪) 大型 底栖动物群落进行了为期一周年的调查研究, 探讨 4 种蜉蝣优势种的种群动态和周年生产量及动态 变化.

\section{1 工作方法}

\section{1 采样点和采样时间}

胡家溪是长江支流清江的一条二级河流, 位于长阳土家族自治县境内 $\left(30^{\circ} 27^{\prime} \sim 30^{\circ} 28^{\prime} \mathrm{N}, 111^{\circ} 03^{\prime} \sim\right.$ $111^{\circ} 35^{\prime} \mathrm{E}$ ), 流域面积约 $50 \mathrm{~km}^{2}$, 于胡家窝附近注人清江, 距离隔河岩水电站约 $8 \mathrm{~km}$. 胡家溪两侧为山脉, 植 被覆盖率较高, 郁闭度较高, 河两岸着生灌木和杂草. 流域地区为典型亚热带气候, 多年平均降水量约 $1460 \mathrm{~mm}$, 全年水温变化范围在 $10 \sim 28^{\circ} \mathrm{C}$ 之间.

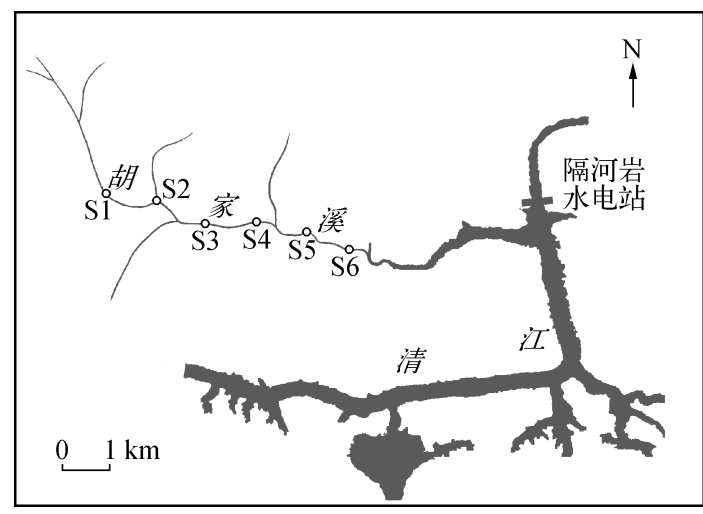

图 1 胡家溪及采样点分布

Fig. 1 Distribution of sampling sites at Hujiaxi Stream

根据河流微生境的类型 (底质、水流速度、水生 植物等), 从上游至下游选择了 6 个采样点 (图 1), 分别是: $\mathrm{S} 1$ : 河边生长着茂密的灌木和杂草, 河面较 窄, 约 1 2 $\mathrm{m}$ 宽, 且部分被遮蔽着, 水流较缓, 底质 为粗沙; $\mathrm{S} 2$ : 有一支流汇人其中, 河面较窄, 约 $2 \mathrm{~m}$ 宽, 水流较缓, 河底以细沙为主; $\mathrm{S} 3$ : 在一个落差约 $0.5 \mathrm{~m}$ 的小瀑布下方, 水流较急, 河底以布满了苔藓 的巨石为主, 附近 $10 \mathrm{~m}$ 处有一片小竹林; $\mathrm{S} 4$ : 位于 一堰塘下游浅水区, 地势平缓, 水流缓慢, 底质为石 灰岩, 河边杂草较少; 55 : 河面变宽, 河宽约 $3 \mathrm{~m}$, 河 底以卵石为主, 腐殖质较多, 水流较急; $\mathrm{S} 6$ : 位于河 口, 一排污口的下游, 河宽约 $4 \mathrm{~m}$, 水流较急, 河底碎 石较多. 胡家溪河水的理化性质见表 1. 采样时间一 般为每月的 5 日左右, 为期一年, 即 2006 年 4 月至 2007 年 3 月.

表 1 胡家溪的理化性质

Tab. 1 Physico-chemical characteristics of Hujiaxi Stream

\begin{tabular}{|c|c|c|c|c|c|c|c|c|c|}
\hline $\mathrm{pH}$ & $\begin{array}{l}\text { 悬浮物/ } \\
(\mathrm{mg} / \mathrm{L})\end{array}$ & $\begin{array}{l}\text { 总磷/ } \\
(\mathrm{mg} / \mathrm{L})\end{array}$ & $\begin{array}{l}\text { 总氮/ } \\
(\mathrm{mg} / \mathrm{L})\end{array}$ & $\begin{array}{l}\text { 氨氮/ } \\
(\mathrm{mg} / \mathrm{L})\end{array}$ & $\begin{array}{c}\text { 亚硝酸盐氮/ } \\
(\mathrm{mg} / \mathrm{L})\end{array}$ & $\begin{array}{c}\text { 硝酸盐氮/ } \\
(\mathrm{mg} / \mathrm{L})\end{array}$ & $\begin{array}{l}\text { 溶解氧/ } \\
(\mathrm{mg} / \mathrm{L})\end{array}$ & $\begin{array}{c}\text { 高锰酸盐指数/ } \\
(\mathrm{mg} / \mathrm{L})\end{array}$ & $\begin{array}{c}\text { BOD/ } \\
(\mathrm{mg} / \mathrm{L})\end{array}$ \\
\hline $\begin{array}{c}7.4 \pm \\
0.8\end{array}$ & $\begin{array}{c}5.3 \pm \\
0.9\end{array}$ & $\begin{array}{c}0.064 \pm \\
0.009\end{array}$ & $\begin{array}{c}1.362 \pm \\
0.230\end{array}$ & $\begin{array}{c}0.89 \pm \\
0.08\end{array}$ & $\begin{array}{c}0.175 \pm \\
0.035\end{array}$ & $\begin{array}{c}0.45 \pm \\
0.05\end{array}$ & $\begin{array}{c}6.28 \pm \\
0.68\end{array}$ & $\begin{array}{c}4.20 \pm \\
0.85\end{array}$ & $\begin{array}{c}3.30 \pm \\
0.53\end{array}$ \\
\hline
\end{tabular}

\section{2 标本采集及处理}

定量采集使用 60 目的 D 型网和 Surber 网,每个采样点面积约为 $30 \mathrm{~cm} \times 30 \mathrm{~cm}$, 每样点 1 次, 1 个重复. 样品经 60 目铜篮篮洗后, 置于白色解剖盘中分检, 标本用 $10 \%$ 的福尔马林固定保存.

\section{3 生活史}

根据各月种群的龄期分布频率了解其发育动态, 从而推知种群生活史状况 ${ }^{[7-8]}$. 


\section{4 生产量的测算}

采用体长 (龄期) 频率法 ( size (instar) -frequency method) 测算其周年生产量 ${ }^{[9-10]}$, 其计算公式为:

$$
P=\sum_{j=1}^{i}\left(N_{j}-N_{j+1}\right)\left(W_{j} \cdot W_{j+1}\right)^{1 / 2} \times 365 / C P I
$$

式中, $C P I$ (Cohort Production Interval) 为一个同龄组完成生活史所需时间. 在此, 忽略矫正系数 $i(P e / P)$, 令其 等于 1 , 原因是 $P e / P$ 在实际计算中非常困难,而且造成的误差极小 ${ }^{[11]}$.

\section{5 生产量的动态}

按照 Benke 等 ${ }^{[8]}$ 的方法, 测算各同龄组的 $P / B$ (生产量/生物量) 系数, 然后计算出日 $P / B$ 系数,再根据 各采样时段的现存量, 求出各时段的生产量动态. 生产量的时间重叠采用比例相似系数 (Proportional Similarity index, $P S)^{[12]}$ 表示为:

$$
P S_{a b}=\sum_{i=1}^{n} \min \left(P_{a, i}, P_{b, i}\right)
$$

式中, $P S_{a b}$ 表示 $a 、 b$ 两物种比例相似性, $n$ 表示采样间隔数, $P_{a, i}$ 表示 $a$ 物种第 $i$ 间隔生产量所占比例, $P_{b, i}$ 表 示 $b$ 物种第 $i$ 间隔生产量所占比例.

\section{2 结果}

\section{1 种群动态}

小裳蜉种群密度和生物量 (湿重, 以下同) 的变化趋势基本相同, 周年平均值分别为 $407 \mathrm{ind} . / \mathrm{m}^{2}$ 和 $1.00 \mathrm{~g} / \mathrm{m}^{2}$. 密度在 2006 年 4 月达到峰值 (1156 ind. $\left./ \mathrm{m}^{2}\right)$, 生物量则在 2006 年 5 月达到峰值 $\left(2.40 \mathrm{~g} / \mathrm{m}^{2}\right)$, 密 度和生物量在其它各月均保持较低水平 (图 2a).

扁蜉种群密度和生物量的变化趋势较为不同, 周年平均值分别为 $150 \mathrm{ind} . / \mathrm{m}^{2}$ 和 $0.37 \mathrm{~g} / \mathrm{m}^{2}$. 密度在 2007 年 3 月出现峰值 $\left(256 \mathrm{ind} . / \mathrm{m}^{2}\right)$, 明显高于其它各月; 生物量则出现两次峰值, 分别在 4 月 $\left(0.94 \mathrm{~g} / \mathrm{m}^{2}\right)$ 和次 年 3 月 $\left(1.05 \mathrm{~g} / \mathrm{m}^{2}\right)$, 其中次年 3 月为主峰值 (图 $2 \mathrm{~b}$ ).
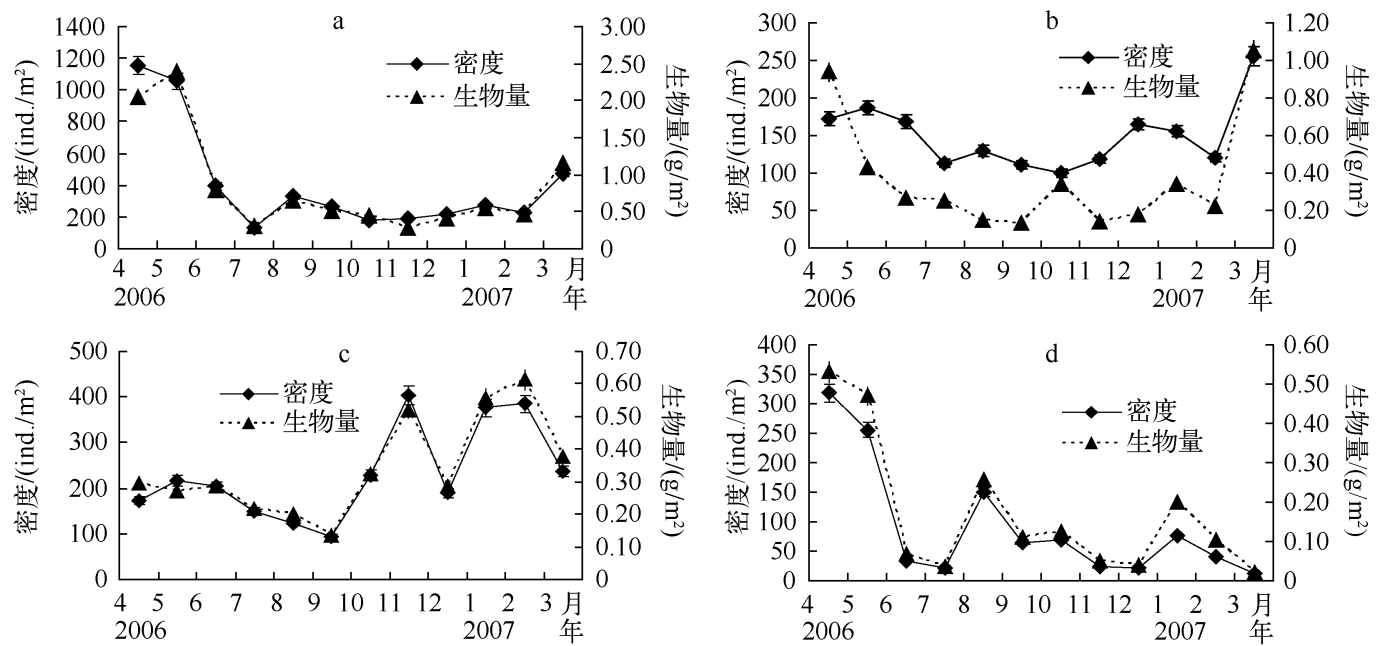

图 2 胡家溪小裳蜉 $(a)$ 、扁蜉 $(b)$ 、四节蜉 $(c)$ 和细蜉 $(d)$ 的现存量周年动态

Fig. 2 Annual variations of standing stock of Leptophlebia sp. (a), Electrogena sp. (b), Indobaetis sp. (c) and Caenis sp. (d) in Hujiaxi Stream

四节蜉种群密度和生物量的变化较为一致. 密度出现两次峰值, 即在 11 月达到主峰值 ( $\left.404 \mathrm{ind} . / \mathrm{m}^{2}\right)$, 次年 2 月达到次峰值 $\left(385 \mathrm{ind} . / \mathrm{m}^{2}\right)$; 生物量也出现两次峰值, 分别在次年 1 月 $\left(0.56 \mathrm{~g} / \mathrm{m}^{2}\right)$ 和次年 2 月 
$\left(0.61 \mathrm{~g} / \mathrm{m}^{2}\right)$. 四节蜉密度和生物量的周年平均值分别为 232 ind. $/ \mathrm{m}^{2}, 0.30 \mathrm{~g} / \mathrm{m}^{2}$ (图 $2 \mathrm{c}$ ).

细蜉种群密度和生物量的变化几乎完全同步, 密度出现三次峰值, 即在 4 月达到主峰值 $\left(319 \mathrm{ind} . / \mathrm{m}^{2}\right)$, 8 月达到次峰值 $\left(150 \mathrm{ind} . / \mathrm{m}^{2}\right)$, 次年 1 月份达到第三个峰值 $\left(76 \mathrm{ind} . / \mathrm{m}^{2}\right)$; 生物量的三次峰值分别为 0.53 、 $0.26 、 0.20 \mathrm{~g} / \mathrm{m}^{2}$. 细蜉密度和生物量的周年平均值分别为 91 ind. $/ \mathrm{m}^{2} 、 0.17 \mathrm{~g} / \mathrm{m}^{2}$ (图 $2 \mathrm{~d}$ ).

\section{2 生活史}

小裳蜉成虫羽化主要发生在 $8 、 10$ 月和次年的 $1 、 2 、 3$ 月, 随后交配产卵. 繁殖发生在 $4 、 9$ 月和 $10 、$ 11 月, 11 月出生的个体由于冬天气温较低生长发育缓慢, 至次年 $2 、 3$ 月开始生长较快. 因此, 可判断 小裳蜉一周年有三个世代: 4-8 月、7-10 月为一个世代, 11 月至次年 3 月为一个世代, 世代有重叠 ( 图 3a).

扁蜉成虫羽化时间为 4 月和次年 $2 、 3$ 月,随后交配产卵, 繁殖主要发生在 $5 、 6$ 月和 $8 、 9 、 11$ 月, 因此亦可 划分为三个世代,即 5-7 月为一个世代、8-10 月为一个世代、11 月至次年 4 月为一个世代(图 $3 b$ ).

四节蜉成虫羽化时间为 4 月、10 月和次年 $2 、 3$ 月, 繁殖主要发生在 $5 、 11 、 12$ 月和次年 $1 、 2$ 月, 因此可以 粗略划分三个世代, 即 5-8 月为一个世代, $8-10$ 月, 11 月至次年 4 月为一个世代, 但世代间存在重叠现象 ( 图 3c).

细蜉成虫的羽化主要发生在 4 月、5 月和次年 1 月,随后交配产卵. 繁殖主要发生 $4 、 7$ 和 12 月. 由此可 粗略划分出两个世代,即 6-11 月一个世代,12 月至次年 5 月一个世代( 图 3d).

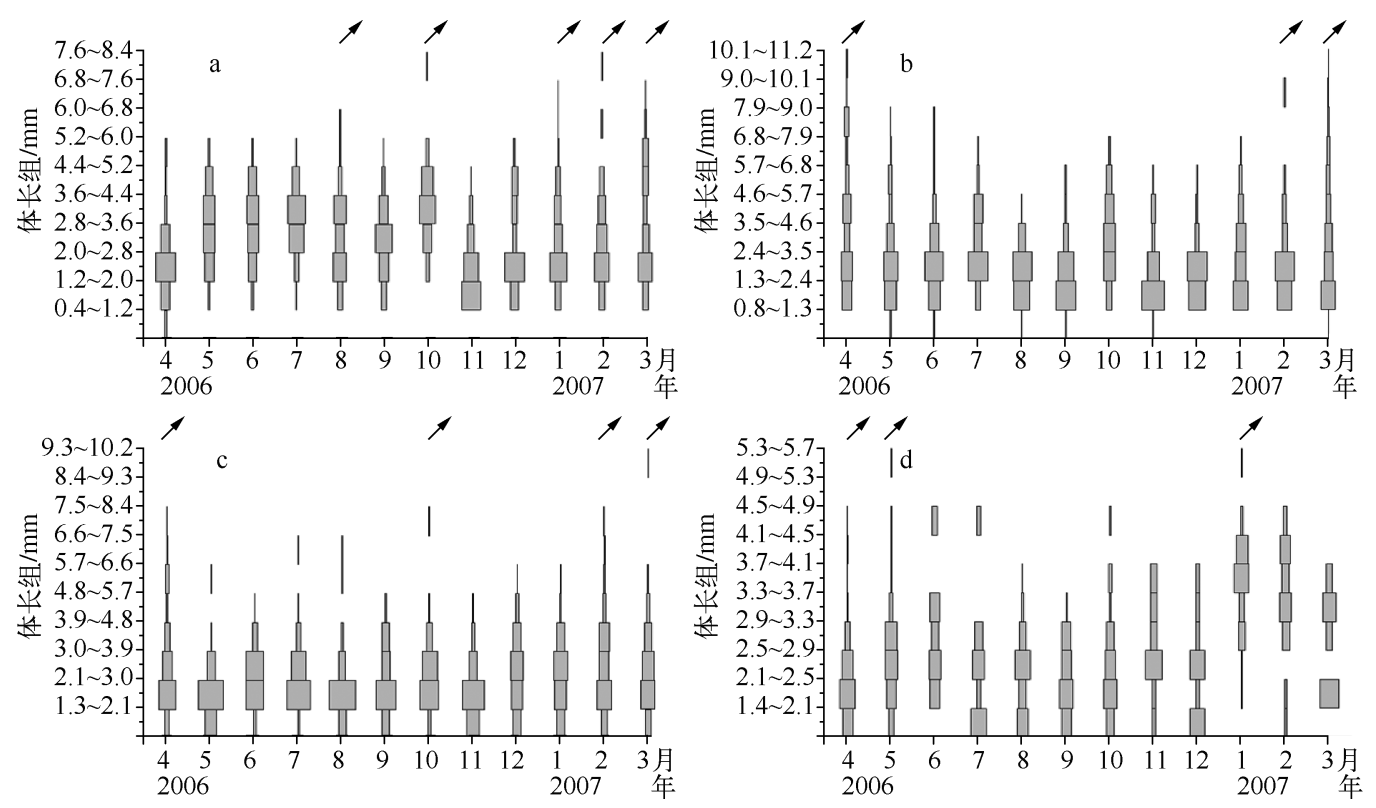

图 3 胡家溪小裳蜉 $(\mathrm{a})$ 、扁蜉 $(\mathrm{b})$ 、四节蜉 $(\mathrm{c})$ 和细蜉 $(\mathrm{d})$ 的种群体长频率分布逐月动态 ( 表示幼虫羽化)

Fig. 3 Monthly size-frequency distributions of Leptophlebia sp. (a), Electrogena sp. (b), Indobaetis sp. (c) and Caenis sp. (d) in Hujiaxi Stream

\section{3 周年生产量}

2.3.1 小裳蜉的周年生产量 小裳蜉一年完成三个世代, 其周年生产量为其同龄群的 3 倍. 小裳蜉的同龄群 生产量为 $147.14 \mathrm{~g} / \mathrm{m}^{2}$, 现存量为 $30.87 \mathrm{~g} / \mathrm{m}^{2}$, 周年 $P / B$ 系数为 14.3 . 周年生产量湿重为 $441.42 \mathrm{~g} / \mathrm{m}^{2}$, 换算成 干重 ${ }^{[13]}$ 为 $88.28 \mathrm{~g} / \mathrm{m}^{2}$ (表 2 ).

2.3 .2 扁蜉的周年生产量 扁蜉一年亦完成三个世代, 周年生产量为其同龄群生产量的 3 倍. 扁蜉同龄 群生产量为 $144.96 \mathrm{~g} / \mathrm{m}^{2}$, 现存量为 $24.77 \mathrm{~g} / \mathrm{m}^{2}$, 周年 $P / B$ 系数为 17.6 ; 周年生产量湿重为 $434.88 \mathrm{~g} / \mathrm{m}^{2}$, 换 算成干重为 $86.98 \mathrm{~g} / \mathrm{m}^{2}$ (表 3 ). 
表 2 胡家溪小裳蜉的周年生产量

Tab. 2 Annual production of Leptophlebia sp. in Hujiaxi Stream

\begin{tabular}{lccccccc}
\hline 体长组 $/ \mathrm{mm}$ & $\begin{array}{c}\text { 密度/ } \\
\left(\mathrm{ind} . / \mathrm{m}^{2}\right)\end{array}$ & $\begin{array}{c}\text { 体均重/ } \\
\mathrm{mg}\end{array}$ & $\begin{array}{c}\text { 生物量/ } \\
\left(\mathrm{g} / \mathrm{m}^{2}\right)\end{array}$ & $\begin{array}{c}\text { 减员数/ } \\
\left(\mathrm{ind} / \mathrm{m}^{2}\right)\end{array}$ & $\begin{array}{c}\text { 减员个体均重/ } \\
\mathrm{mg}\end{array}$ & $\begin{array}{c}\text { 损失量/ } \\
\left(\mathrm{g} / \mathrm{m}^{2}\right)\end{array}$ & $\begin{array}{c}\text { 生产量/ } \\
\left(\mathrm{g} / \mathrm{m}^{2}\right)\end{array}$ \\
\hline $0.4 \sim 1.2$ & 388.89 & 0.03 & 0.0117 & -3133.33 & 0.11 & -0.34 & -3.45 \\
$1.2 \sim 2.0$ & 3522.22 & 0.22 & 0.7749 & -5244.4 & 0.31 & -1.65 & -16.50 \\
$2.0 \sim 2.8$ & 8766.67 & 0.45 & 3.9450 & 2166.67 & 0.63 & 1.36 & 13.56 \\
$2.8 \sim 3.6$ & 6600.00 & 0.87 & 5.7420 & 1133.33 & 1.14 & 1.29 & 12.90 \\
$3.6 \sim 4.4$ & 5466.67 & 1.49 & 8.1453 & 2166.67 & 1.81 & 3.92 & 39.23 \\
$4.4 \sim 5.2$ & 3300.00 & 2.20 & 7.2600 & 2200.00 & 2.63 & 5.78 & 57.82 \\
$5.2 \sim 6.0$ & 1100.00 & 3.14 & 3.4540 & 888.89 & 3.68 & 3.27 & 32.74 \\
$6.0 \sim 6.8$ & 211.11 & 4.32 & 0.9120 & 144.44 & 4.32 & 0.62 & 6.24 \\
$6.8 \sim 7.6$ & 66.67 & 5.69 & 0.3800 & 33.33 & 6.46 & 0.22 & 2.15 \\
$7.6 \sim 8.4$ & 33.33 & 7.33 & 0.2400 & 33.33 & 7.33 & 0.24 & 2.44 \\
\hline
\end{tabular}

表 3 胡家溪扁蜉的周年生产量

Tab. 3 Annual production of Electrogena sp. in Hujiaxi Stream

\begin{tabular}{rrrrrrrr}
\hline 体长组 $/ \mathrm{mm}$ & $\begin{array}{c}\text { 密度/ } \\
\left(\mathrm{ind} . / \mathrm{m}^{2}\right)\end{array}$ & $\begin{array}{c}\text { 体均重/ } \\
\mathrm{mg}\end{array}$ & $\begin{array}{c}\text { 生物量/ } \\
\left(\mathrm{g} / \mathrm{m}^{2}\right)\end{array}$ & $\begin{array}{c}\text { 减员数/ } \\
\left(\mathrm{ind} . / \mathrm{m}^{2}\right)\end{array}$ & $\begin{array}{r}\text { 减员个体均重/ } \\
\mathrm{mg}\end{array}$ & $\begin{array}{r}\text { 损失量/ } \\
\left(\mathrm{g} / \mathrm{m}^{2}\right)\end{array}$ & $\begin{array}{c}\text { 生产量/ } \\
\left(\mathrm{g} / \mathrm{m}^{2}\right)\end{array}$ \\
\hline $0.7 \sim 1.3$ & 55.56 & 0.07 & 0.0039 & -3044.44 & 0.11 & -0.33 & -3.35 \\
$1.3 \sim 2.4$ & 3100.00 & 0.35 & 1.0850 & -177.78 & 0.58 & -0.10 & -1.03 \\
$2.4 \sim 3.5$ & 3277.78 & 0.95 & 3.1139 & 1788.89 & 1.52 & 2.72 & 27.18 \\
$3.5 \sim 4.6$ & 1488.89 & 2.43 & 3.6180 & 488.89 & 3.32 & 1.62 & 16.24 \\
$4.6 \sim 5.7$ & 1000.00 & 4.54 & 4.5400 & 511.11 & 5.90 & 3.02 & 30.18 \\
$5.7 \sim 6.8$ & 488.89 & 7.68 & 3.7547 & 277.78 & 9.80 & 2.72 & 27.22 \\
$6.8 \sim 7.9$ & 211.11 & 12.50 & 2.6389 & 44.44 & 15.56 & 0.69 & 6.92 \\
$7.9 \sim 9.0$ & 166.67 & 19.38 & 3.2300 & 111.11 & 19.38 & 2.15 & 21.53 \\
$9.0 \sim 10.1$ & 55.56 & 26.97 & 1.4983 & 22.22 & 32.30 & 0.72 & 7.18 \\
$10.1 \sim 11.2$ & 33.33 & 38.68 & 1.2893 & 33.33 & 38.68 & 1.29 & 12.89 \\
\hline
\end{tabular}

2.3.3 四节蜉的周年生产量 四节蜉一年完成三个世代,周年生产量为其同龄群生产量的 3 倍. 四节蜉同龄 群生产量为 $249.07 \mathrm{~g} / \mathrm{m}^{2}$, 现存量为 $49.72 \mathrm{~g} / \mathrm{m}^{2}$, 周年 $P / B$ 系数为 15.03 . 则周年生产量湿重为 $747.21 \mathrm{~g} / \mathrm{m}^{2}$, 换算成干重为 $149.44 \mathrm{~g} / \mathrm{m}^{2}$ (表 4).

2.3.4 细蜉的周年生产量 细蜉一年完成两个世代, 周年生产量为其同龄群生产量的 2 倍. 细蜉同龄群 生产量为 $20.26 \mathrm{~g} / \mathrm{m}^{2}$, 现存量为 $5.66 \mathrm{~g} / \mathrm{m}^{2}$, 周年 $P / B$ 系数为 7.20 , 周年生产量湿重为 $40.52 \mathrm{~g} / \mathrm{m}^{2}$, 换 算成干重为 $8.10 \mathrm{~g} / \mathrm{m}^{2}$ (表 5 ).

\section{4 生产量动态}

4 种蜉蝣优势种小裳蜉、扁蜉、四节蜉、细蜉的生产量动态如图 10 所示. 4 种蜉蝣的生产量时间重叠比 例相似系数均较高, 除细蜉和四节蜉为 0.55 以外, 其余均大于 0.60 , 细蜉和小裳蜉、扁蜉和小裳蜉的相似性 系数甚至分别高达 0.81 和 0.79 (表 6 ). 可见, 4 种蜉蝣的生产量时间重叠性较高, 这可能与 4 种蜉蝣的生境 以及食物资源较为相近有关. 
表 4 胡家溪四节蜉的周年生产量

Tab. 4 Annual production of Indobaetis sp. in Hujiaxi Stream

\begin{tabular}{cccccccc}
\hline $\begin{array}{c}\text { 体长组/ } \\
(\mathrm{mm})\end{array}$ & $\begin{array}{c}\text { 密度/ } \\
\left(\mathrm{ind} . / \mathrm{m}^{2}\right)\end{array}$ & $\begin{array}{c}\text { 体均重/ } \\
\mathrm{mg}\end{array}$ & $\begin{array}{c}\text { 生物量/ } \\
\left(\mathrm{g} / \mathrm{m}^{2}\right)\end{array}$ & $\begin{array}{r}\text { 减员数/ } \\
\left(\mathrm{ind} / \mathrm{m}^{2}\right)\end{array}$ & $\begin{array}{c}\text { 减员个体均重/ } \\
\mathrm{mg}\end{array}$ & $\begin{array}{r}\text { 损失量/ } \\
\left(\mathrm{g} / \mathrm{m}^{2}\right)\end{array}$ & $\begin{array}{c}\text { 生产量/ } \\
\left(\mathrm{g} / \mathrm{m}^{2}\right)\end{array}$ \\
\hline $1.2 \sim 2.1$ & 2511.11 & 1.84 & 4.6204 & -3911.11 & 0.11 & -0.43 & -4.30 \\
$2.1 \sim 3.0$ & 6422.22 & 2.55 & 16.3767 & 2111.11 & 2.93 & 6.18 & 61.79 \\
$3.0 \sim 3.9$ & 4311.11 & 3.36 & 14.4853 & 2177.78 & 3.80 & 8.27 & 82.68 \\
$3.9 \sim 4.8$ & 2133.33 & 4.29 & 9.1520 & 1644.44 & 4.70 & 7.74 & 77.37 \\
$4.8 \sim 5.7$ & 488.89 & 5.16 & 2.5227 & 211.11 & 5.61 & 1.19 & 11.85 \\
$5.7 \sim 6.6$ & 277.78 & 6.11 & 1.6972 & 244.44 & 6.43 & 1.57 & 15.72 \\
$6.6 \sim 7.5$ & 33.33 & 6.77 & 0.2257 & -33.33 & 7.30 & -0.24 & -2.43 \\
$7.5 \sim 8.4$ & 66.67 & 7.88 & 0.5253 & 66.67 & 7.88 & 0.53 & 5.25 \\
$8.4 \sim 9.3$ & 0 & 0 & 0 & -11.11 & 0 & 0 & 0 \\
$9.3 \sim 10.2$ & 11.11 & 10.19 & 0.1132 & 11.11 & 10.19 & 0.11 & 1.13 \\
\hline
\end{tabular}

表 5 胡家溪细蜉的周年生产量

Tab. 5 Annual production of Caenis sp. in Hujiaxi Stream

\begin{tabular}{ccccccrc}
\hline $\begin{array}{c}\text { 体长组/ } \\
\mathrm{mm}\end{array}$ & $\begin{array}{c}\text { 密度/ } \\
\left(\mathrm{ind} . / \mathrm{m}^{2}\right)\end{array}$ & $\begin{array}{c}\text { 体均重/ } \\
\mathrm{mg}\end{array}$ & $\begin{array}{c}\text { 生物量/ } \\
\left(\mathrm{g} / \mathrm{m}^{2}\right)\end{array}$ & $\begin{array}{r}\text { 减员数/ } \\
\left(\mathrm{ind} . / \mathrm{m}^{2}\right)\end{array}$ & $\begin{array}{r}\text { 减员个体均重/ } \\
\mathrm{mg}\end{array}$ & $\begin{array}{r}\text { 损失量/ } \\
\left(\mathrm{g} / \mathrm{m}^{2}\right)\end{array}$ & $\begin{array}{c}\text { 生产量/ } \\
\left(\mathrm{g} / \mathrm{m}^{2}\right)\end{array}$ \\
\hline $1.4 \sim 2.1$ & 1066.67 & 0.40 & 0.4267 & -422.22 & 0.11 & -0.05 & -0.46 \\
$2.1 \sim 2.5$ & 1488.89 & 0.60 & 0.8933 & -77.78 & 0.69 & -0.05 & -0.54 \\
$2.5 \sim 2.9$ & 1566.67 & 0.80 & 1.2533 & 477.78 & 0.89 & 0.43 & 4.27 \\
$2.9 \sim 3.3$ & 1088.89 & 1.00 & 1.0889 & 633.33 & 1.13 & 0.72 & 7.17 \\
$3.3 \sim 3.7$ & 455.56 & 1.28 & 0.5831 & 133.33 & 1.46 & 0.19 & 1.94 \\
$3.7 \sim 4.1$ & 322.22 & 1.66 & 0.5349 & 55.56 & 1.83 & 0.10 & 1.01 \\
$4.1 \sim 4.5$ & 266.67 & 2.01 & 0.5360 & 155.56 & 2.20 & 0.34 & 3.42 \\
$4.5 \sim 4.9$ & 111.11 & 2.40 & 0.2667 & 111.11 & 2.40 & 0.27 & 2.67 \\
$4.9 \sim 5.3$ & 0 & 0 & 0 & -22.22 & 0 & 0 & 0 \\
$5.3 \sim 5.7$ & 22.22 & 3.54 & 0.0787 & 22.22 & 3.54 & 0.08 & 0.79 \\
\hline
\end{tabular}

\section{3 讨论}

本研究地点位于亚热带地区, 4 种蜉蝣中, 细蜉为一年两代, 小裳蜉、扁蜉和四节蜉均为一年三代, 与文 献报道的亚热带地区蜉蝣一般为一年 $1 \sim 3$ 个世代的规律基本吻合 ${ }^{[14-17]}$.

就生产量而言, 本研究中的 4 种蜉蝣平均周年生产量换算成干重分别为: 小裳蜉 $88.28 \mathrm{~g} / \mathrm{m}^{2}$ 、扁蜉 $86.98 \mathrm{~g} / \mathrm{m}^{2}$ 、四节蜉 $149.44 \mathrm{~g} / \mathrm{m}^{2} 、$ 细蜉 $8.10 \mathrm{~g} / \mathrm{m}^{2} ; 4$ 种蜉蝣平均周年 $P / B$ 系数分别为 $14.3 、 17.6 、 15.0 、 7.2$.

表 6 胡家溪蜉蝣优势种间的相似性系数

Tab. 6 Proportional similarity between mayfly dominant species in Hujiaxi Stream

\begin{tabular}{ccccc}
\hline 物种名 & 小裳蜉 & 扁蜉 & 四节蜉 & 细蜉 \\
\hline 小裳蜉 & 1 & & & \\
扁蜉 & 0.79 & 1 & & \\
四节蜉 & 0.63 & 0.68 & 1 & \\
细蜉 & 0.81 & 0.67 & 0.54 & 1 \\
\hline
\end{tabular}

4 种蜉蝣的生产量与已报道的其他河流蜉蝣的生产量 相比较为适中 ${ }^{[14-27]}$ (表 7). 但按照 Huryn 等 $^{[28]}$ 对溪流 底栖动物生产量的统计, 这些蜉蝣的生产量除细蜉小 于 $100 \mathrm{~g} / \mathrm{m}^{2}$, 四节蜉的生产量大于 $100 \mathrm{~g} / \mathrm{m}^{2}$, 其余两种 接近于“高生产量” 的范围 $\left(100 \sim 300 \mathrm{~g} / \mathrm{m}^{2}\right.$ ( DW ) ), 这 与胡家溪的总磷含量较高 $(0.064 \mathrm{mg} / \mathrm{L})$, 藻类生长 较多、较快, 且胡家溪的郁闭度较高, 植物碎屑充足 有关. 另一方面, 蜉蝣的捕食者 (鱼类、蟹类) 相对较 少, 捕食压力也小. 4 种蜉蝣的 $P / B$ 系数与其他河流 的相比较为适中, 特别与同地区的叹气沟河蜉蝣的 
$P / B$ 系数较为接近, 这也说明了在环境条件相似的区域,物种的 $P / B$ 系数比较稳定.

表 7 不同水体蜉蝣生产力和 $P / B$ 系数比较

Tab. 7 Secondary production and $P / B$ ratio of mayfly species

\begin{tabular}{|c|c|c|c|c|}
\hline 物种 & $\begin{array}{c}\text { 生产量 }(P) / \\
\left(\mathrm{g}(\mathrm{DW}) / \mathrm{m}^{2}\right)\end{array}$ & $\begin{array}{l}P / B \\
\text { 系数 }\end{array}$ & 生境 & 来源 \\
\hline Caenis luctuosa & 6.350 & 15.98 & Mediterranean semiarid stream(Murcia, Spain) & 文献 $[16]$ \\
\hline C. amica & 0.445 & 13.00 & Experimental ponds ( Virginia, USA) & 文献 $[19]$ \\
\hline C. amica & 0.676 & 12.72 & Experimental stream(Alabama, USA) & 文献 $[20]$ \\
\hline Caenis spp. & 0.082 & 59.10 & Humid/mesic plains stream( Georgia, USA) & 文献 $[21]$ \\
\hline C. rivulorum & 0.030 & - & Experimental stream( Dorset, UK) & 文献 [22] \\
\hline C. luctuosa & 0.076 & 7.90 & Reach 7 (Northern Spain) & 文献 $[17]$ \\
\hline C. luctuosa & 0.093 & 7.10 & Reach 9(Northern Spain) & 文献 $[17]$ \\
\hline C. amica & 0.400 & 13.00 & Experimental pond (Virginia, USA) & 文献 $[19]$ \\
\hline C. horaria & 0.539 & 10.20 & Woodland pond( Geneva, Switzerland) & 文献 $[23]$ \\
\hline C. simulans & 0.560 & 4.40 & Plains stream( Minnesota, USA) & 文献 [24] \\
\hline C. luctuosa & 0.082 & 23.70 & Semiarid stream( Murcia, Spain) & 文献 $[25]$ \\
\hline Afronurus sp. & 2.406 & 34.70 & Shing Mun River (Hong Kong) & 文献 $[2]$ \\
\hline Cinygmina sp. & 1.198 & 33.00 & Shing Mun River (Hong Kong) & 文献 $[2]$ \\
\hline Procloeon sp. & 0.099 & 99.50 & Shing Mun River (Hong Kong) & 文献 $[2]$ \\
\hline Baetiella pseudofrequenta & 0.143 & 95.40 & Shing Mun River (Hong Kong) & 文献 $[2]$ \\
\hline Choroterpes sp. & 0.226 & 57.80 & Shing Mun River (Hong Kong) & 文献 $[2]$ \\
\hline H. limbata & 4.688 & 4.09 & in Blackwater Creek ( central Florida,USA) & 文献 $[25]$ \\
\hline H. limbata & 0.003 & 4.59 & in Rock Springs Run ( central Florida, USA) & 文献 $[25]$ \\
\hline Hb. lauta & 0.199 & 9.6 & A Northern Spain stream & 文献 [26] \\
\hline Hd. confusa & 0.413 & 5.7 & A Northern Spain stream & 文献 [26] \\
\hline Leptophlebia sp. & 7.303 & 11.4 & 中国湖北黑竹冲河 & 文献 $[15]$ \\
\hline Ephemera sp. & 14.598 & 11.8 & 中国湖北黑竹冲河 & 文献 $[15]$ \\
\hline Caenis sp. & 5.064 & 6.4 & 中国湖北叹气沟河 & 文献 [27] \\
\hline Choroterpes sp. & 9.696 & 7.1 & 中国湖北叹气沟河 & 文献 [27] \\
\hline Indobaetis sp. & 52.812 & 17.0 & 中国湖北叹气沟河 & 文献 [27] \\
\hline Electrogena sp. & 112.020 & 11.5 & 中国湖北叹气沟河 & 文献 [27] \\
\hline Leptophlebia sp. & 88.280 & 14.3 & 中国湖北胡家溪 & 本研究 \\
\hline Electrogena sp. & 86.980 & 17.6 & 中国湖北胡家溪 & 本研究 \\
\hline Indobaetis sp. & 149.440 & 15.0 & 中国湖北胡家溪 & 本研究 \\
\hline Caenis sp. & 8.100 & 7.2 & 中国湖北胡家溪 & 本研究 \\
\hline
\end{tabular}

本研究的 4 种蜉蝣优势种之间比较, 二翼蜉的周年生产量最大, 这可能与二翼蜉的种群密度、生物量最 大有关, 且其 $P / B$ 系数也达到了 15.0 , 仅次于扁蜉的 $P / B$ 系数 17.6. 细蜉的周年生产量最小, 明显小于其余 三种, 可能与其种群密度较小有关. 就 $P / B$ 系数而言, 扁蜉的最大, 这可能与胡家溪扁蜉个体相对较小 $(75 \%$ 以上的个体体长在 $1.3 \sim 4.6 \mathrm{~mm}$ 范围内), 保持较快生长速率 (周转率) 能使得其更加适应胡家溪的生态环 境, 从而在竞争资源中占据优势有关. 小裳蜉有 $80 \%$ 以上的个体的体长在 $2.0 \sim 5.2 \mathrm{~mm}$ 范围内, 在胡家溪的 几种蜉蝣中个体中相对较大, $P / B$ 系数在几种蜉蝣中最小. 这与 Huryn 等报道的溪流水生昆虫 $P / B$ 系数通 常与其个体大小呈反相关关系相一致 ${ }^{[28]}$.

从生产量动态来看, 4 种蜉蝣的生产量除细蜉在 2007 年 3 月的日均生产量较低, 可能与采样误差有关 以外, 其余 3 种蜉蝣在 3、4、5 月生产量均维持在较高水平 (图 4), 这可能由于越冬的一代蜉蝣在春季的大量 繁殖, 种群密度较高, 故导致其生产量较高. 而在 8-10月, 4 种蜉蝣的生产量均较低, 这可能与圆顶华溪蟹 等捕食者在 8-10 月的大量繁殖有关. 有研究表明四节蜉科 Baetidae 的幼虫在有捕食者存在时基本不生长, 

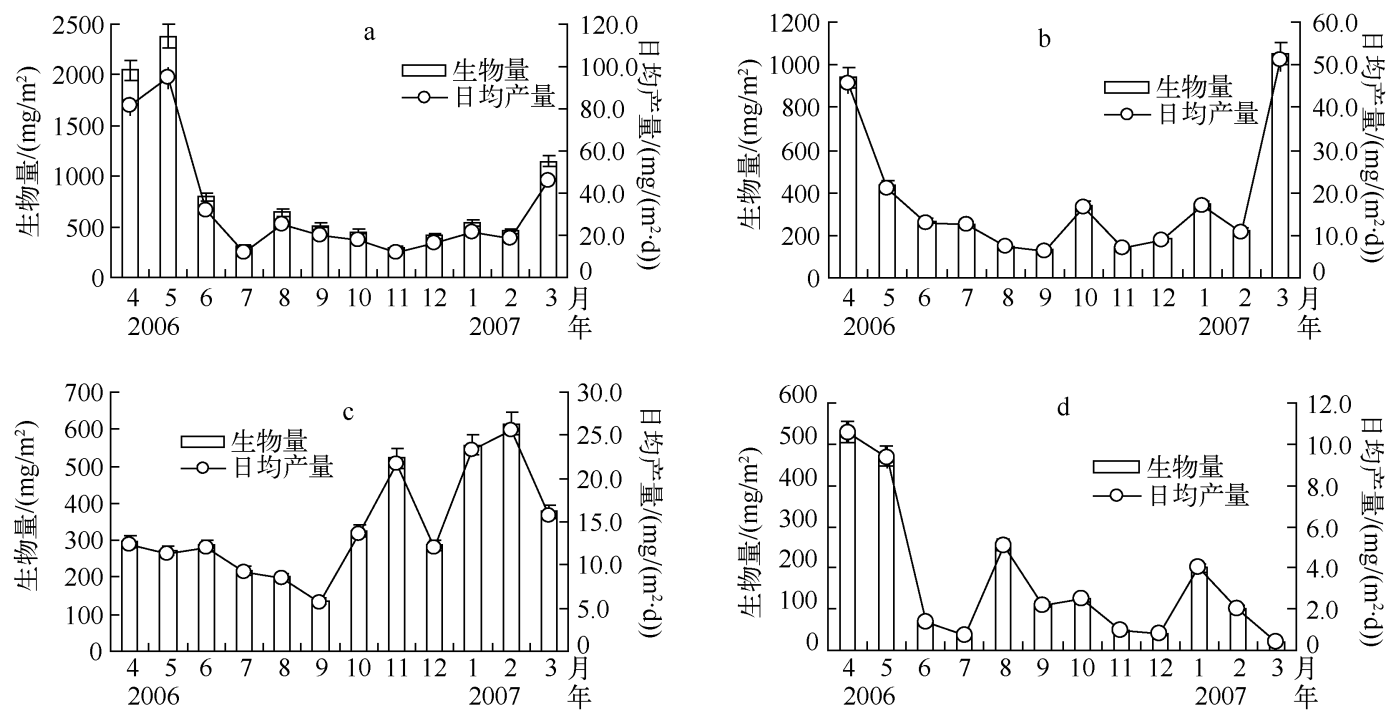

图 4 胡家溪小裳蜉 $(\mathrm{a}) 、$ 扁蜉 $(\mathrm{b}) 、$ 四节蜉 $(\mathrm{c}) 、$ 细蜉 $(\mathrm{d})$ 的日均产量及生产量动态

Fig. 4 Temporal pattern of interval biomass and mean daily production for Leptophlebia sp. (a), Electrogena sp. (b), Indobaetis sp. (c) and Caenis sp. (d) in Hujiaxi Stream

但在没有捕食者存在时, 生物量一周就能增加 $50 \%{ }^{[28]} .4$ 种蜉蝣之间的时间重叠相似性系数除细蜉和四节 蜉的为 0.55 以外,其余均大于 0.60 , 细蜉和小裳蜉、扁蜉和小裳蜉的相似性系数甚至分别高达 $0.81 、 0.79$. 但这仍然在 Benke 报道的范围之内 ${ }^{[21]} .4$ 种蜉蝣之间缺乏时间维度的分离, 可能与这 4 种蜉蝣在全年均有 出现以及它们的多化性、食物资源丰富有关.

\section{4 参考文献}

[ 1 ] Benke AC, Huryn AD. Benthic invertebrate production - facilitating answers to ecological riddles in freshwater ecosystems. J N Am Benthol Soc, 2010, 29(1) :264-285.

[ 2 ] Salas M, Dudgeon D. Life histories, production dynamics and resource utilisation of mayflies (Ephemeroptera) in two tropical Asian forest streams. Freshwater Biol, 2003, 48:485-499.

[ 3 ] Yan Y, Li X. Secondary production and its trophic basis of two species of mayfly in a subtropical stream of China. Int Rev Hydrobiol, 2006, $91(2): 136-147$.

[ 4 ] Huang Y, Yan Y, Li X. Food web structure of benthic macroinvertebrates in a second order stream of the Hanjiang River basin in middle China. J Freshwater Ecol, 2008, 23 (3):421-427.

[ 5 ] Wen F, Jiang J, Deng S et al. Food web and trophic basis of production of the benthic macroinvertebrate community of a subtropical stream (Yangtze River Basin, Middle China). Int Rev Hydrobiol, 2009, 95 (4/5) :395-409.

[6]王备新,杨莲芳. 我国东部底栖无脊椎动物主要分类单元耐污值. 生态学报,2004,24(12):2768-2775.

[ 7 ] Georgian T, Wallace JB. Seasonal production dynamics in a guild of periphyton-grazing insects in a southern Appalachian stream. Ecology, 1983, 64:1236-1248.

[ 8 ] Benke AC, Wallace JB. Trophic basis of production among riverine caddisflies: implications for food web analysis. Ecolo$g y, 1997, \mathbf{7 8}(4): 1132-1145$.

[ 9 ] Hynes HBN, Coleman MJ. A simple method of assessing the annual production of stream benthos. Limnol \& Oceanogr, $1968,13: 569-573$.

[10] Hamilton AL. On estimating annual production. Limnol \& Oceanogr, 1969, 14:771-782.

[11] Menzie CA. A note on the Hynes method of estimating secondary production. Limnol \& Oceanogr, 1980, 25 (4): $770-773$ 
[12] Whittaker RH. Communities and ecosystems. New York: Macmillian, 1975: 103.

[13] 间云君. 浅水湖泊大型底栖动物生态能量学及生产量的研究 [学位论文]. 武汉: 中国科学院水生生物研究 所, 1998 .

[14] Yan Y, Li X. Production and its trophic basis of two mayflies (Leptophlebia sp. and Ephemera sp.) in Heizhuchong Stream in China. J Freshwater Ecol, 2006, 21(2) :301-306.

［15］间云君,李晓宇. 黑竹冲河小裳蜉 (Leptophlebia sp. ) 和蜉蝣 (Ephemera sp. ) 周年生产量. 生态科学, 2006,25 (2) : 97-101.

[16] Perán A, Velasco J, Millán A. Life cycle and secondary production of Caenis luctuosa (Ephemeroptera) in a semiarid stream (Southeast Spain). Hydrobiologia, 1999, 400:187-194.

[17] González JM, Basaguren A, Pozo J. Life history and production of Caenis luctuosa (Burmeister) (Ephemeroptera, Caenidae) in two nearby reaches along a small stream. Hydrobiologia, 2001, 452 :209-215.

[18] Christman VD, Voshell JR. Life history, growth and production of Ephemeroptera in experimental ponds. Ann Entomol Soc Am, 1992,85 : 705-712

[19] Rodgers EB. Production of Caenis (Ephemeroptera: Caenidae) in elevated water temperatures. Freshwat Invertebr Biol, $1982,1: 2-16$

[20] Benke AC, Jacobi DI. Production dynamics and resource utilization of snag-dwelling mayflies in a blackwater river. Ecolo$g y, 1994,75: 1219-1232$.

[21] Welton JS, Ladle M, Bass JAB. Growth and production of five species of Ephemeraptera larvae from an experimental recirculating stream. Freshwater Biol, 1982, 12:103-122.

[22] Oertli B. Leaf litter processing and energy flow through macroinvertebrates in a woodland pond (Switzerland). Oecologia, $1993,96: 466-477$.

[23 ] Macfarlane MB, Waters TF. Annual production by caddisflies and mayflies in a western Minnesota plains stream. Can J Fish Aquat Sci, 1982, $39: 1628-1635$.

[24] Perán A. Ciclos de vida y producción secundaria en un río de características semiáridas ( Río Chícamo, SE de la Peninsula Ibérica) : Elcaso de Caenis luctuosa y Sigara scripta[Dissertation]. Murcia: Universidad de Murcia, 1997.

[25] González JM, Basaguren A, Pozo J. Life history, production and coexistence of two Leptophlebiid mayflies in three sites along a Northern Spain stream. Archiv Hydrobiol, 2003, 158(3) :303-316.

[26] Lobinske RJ, Ali A, Stout IJ. Life history and productivity of Hexagenia limbata (Ephemeroptera: Ephemeridae) and selected physicochemical parameters in two tributaries of the Wekiva river, central Florida. Florida Entomol, 1996, 79(4) : 543-551.

[27］苏华武. 长江流域二级河流大型底栖动物群落结构和生产量的研究 [学位论文]. 武汉: 华中科技大学,2007.

[28] Huryn AD, Wallace JB. Life history and productionof stream insects. Ann Rev Entomol, 2000, 45:83-110. 